\title{
Effect of coitus at term on length of gestation, induction of labor, and mode of delivery.
}

\begin{abstract}
OBJECTIVE: To determine coital incidence at term and to estimate its effect on labor onset and mode of delivery. METHODS: Healthy women with uncomplicated pregnancies and established gestational age were recruited to keep a diary of coital activity from 36 weeks of gestation until birth and to answer a short questionnaire. Two hundred women with complete coital diaries were available for analysis. Outcome measures include coitus, postdate pregnancy (defined as pregnancy beyond the estimated date of confinement), gestational length of at least 41 weeks, labor induction at 41 weeks of gestation, and mode of delivery. RESULTS: Reported sexual intercourse at term was influenced by a woman's perception of coital safety, her ethnicity, and her partner's age. After multivariable logistic regression analysis controlling for the women's ethnicity, education, occupation, perception of coital safety, and partner's age, coitus at term remained independently associated with reductions in postdate pregnancy (adjusted odds ratio [AOR] 0.28, 95\% confidence interval $[\mathrm{CI}] 0.13-0.58$, $\mathrm{P}=.001$ ), gestational length of at least 41 weeks (AOR 0.10, 95\% CI 0.04-0.28, P $<.001$ ), and requirement for labor induction at 41 weeks of gestation (AOR 0.08, 95\% CI 0.03-0.26, P $<.001)$. At 39 weeks of gestation, 5 (95\% CI 3.3-10.3) couples needed to have intercourse to avoid one woman having to undergo labor induction at 41 weeks of gestation. Coitus at term had no significant effect on operative delivery (adjusted $\mathrm{P}=.15$ ). CONCLUSION: Reported sexual intercourse at term was associated with earlier onset of labor and reduced requirement for labor induction at 41 weeks.
\end{abstract}

Keyword: Coitus; Coital incidence; Induction of labour; Lenght of gestation; Mode of delivery. 